\section{$\varepsilon^{2}$}

${ }^{1}$ Department of Medicine, Elmhurst Hospital Center, Icahn School of Medicine at Mount Sinai, New York, New York, USA

${ }^{2}$ Echocardiography Laboratory, Department of Cardiology, Elmhurst Hospital Center, Icahn School of Medicine at Mount Sinai, New York, New York USA

${ }^{3}$ Division of Pulmonary/Critical Care Medicine, Department of Medicine, Elmhurst Hospital

Center, Icahn School of Medicine at Mount Sinai, New York, New York, USA

\section{Correspondence to} Dr Justin Seashore, Mount Sinai Services, Elmhurst Hospital Center, 79-01 Broadway, Elmhurst, NY 11373, USA; justin.seashore@mssm.edu

Received 28 May 2015 Revised 11 September 2015 Accepted 14 September 2015 Published Online First 9 October 2015

\section{CrossMark}

To cite: Seashore JB Silbiger JJ, Epelbaum 0. Thorax 2015;70:

1205-1208.

\title{
Uncovering the diagnosis
}

Justin B Seashore, ${ }^{1}$ Jeffrey J Silbiger, ${ }^{2}$ Oleg Epelbaum ${ }^{3}$

JBS (Medicine Resident): A 63-year-old African-American woman presented to the emergency department with progressive dyspnoea of 2-weeks' duration. She denied chest pain, palpitations or syncope. Her medical history was significant for hypertension and diabetes mellitus. She never smoked and denied occupational exposures. She was born and raised in New York State, taking annual trips to North Carolina.

On examination, she was obese and appeared dyspnoeic. She was afebrile with a blood pressure of $152 / 74 \mathrm{~mm} \mathrm{Hg}$, a heart rate of $98 \mathrm{bpm}$, a respiratory rate of $20 / \mathrm{min}$ and an oxygen saturation of $97 \%$ while breathing ambient air. The jugular venous pulse was elevated. Heart sounds were normal, and there was no murmur, gallop, rub or click. Lungs were clear to auscultation and percussion. There was trace pedal oedema. The remainder of the examination was unremarkable.

Initial laboratory evaluation was notable for a serum glucose level of $260 \mathrm{mg} / \mathrm{dL}$ (normal range $74-126 \mathrm{mg} / \mathrm{dL}$ ). Complete blood count and serial troponin measurements were normal. The ECG revealed sinus tachycardia at $100 \mathrm{bpm}$ with borderline low QRS voltage and rare ectopy (figure 1A). The chest radiograph showed an increase in the cardiac silhouette compared with a film obtained 10 months earlier (figure 1B-D). There was no pulmonary vascular congestion, but tiny pleural effusions were seen on the lateral projection. Echocardiography demonstrated a large pericardial effusion (figure 1E) with diastolic inversion of the right atrial and right ventricular free walls consistent with tamponade physiology. Left ventricular size and function were normal. The patient underwent pericardiocentesis, which yielded $860 \mathrm{~mL}$ of clear yellow, lymphocyte-predominant fluid. Biochemical analysis revealed lactate dehydrogenase - $130 \mathrm{U} / \mathrm{L}$ (serum $276 \mathrm{U} / \mathrm{L}$ ), total protein-5.8 g/dL (serum $7.9 \mathrm{~g} / \mathrm{dL}$ ) and glucose-162 mg/dL.

JJS (Cardiology Consultant): This is a case of a middle-aged woman with multiple coronary artery disease risk factors who presented with subacute dyspnoea. Her physical examination was significant for the presence of a raised jugular venous pulse, increased heart rate and elevated blood pressure. Cardiac aetiology of dyspnoea appears less likely, however, as the lungs were clear and the examination of the heart was unremarkable. Furthermore, there were no ischaemic changes or evidence of prior infarction on the ECG. Borderline low QRS voltage could be indicative of a pericardial effusion but is also seen in obesity. Given the absence of pulmonary vascular congestion on radiography, it seems probable that the enlarged cardiac silhouette on the chest radiograph was indeed due to a pericardial effusion rather than to cardiomegaly. Echocardiography did, in fact, demonstrate a large pericardial effusion as well as normal left ventricular size and systolic function. Notably, diastolic inversion of the right atrial and right ventricular free walls was observed, consistent with elevated intrapericardial pressure. By themselves, these echocardiographic findings are, however, insufficient to diagnose pericardial tamponade.

Pericardial effusions are usually asymptomatic, although dyspnoea can occur when tamponade intervenes. Its presence, particularly when accompanied by a raised jugular venous pulse and tachycardia, as in this patient, is therefore of particular concern. Unfortunately, evaluation for a pulsus paradoxus, which is a highly characteristic finding of pericardial tamponade, was not undertaken. Notwithstanding, proceeding to pericardiocentesis, as was done in this case, seems prudent. While the patient's pericardial fluid was consistent with an exudate using Light's criteria for pleural fluid, this is of little diagnostic value as even physiologic pericardial fluid is exudative using these criteria. ${ }^{1}$ The finding of pericardial fluid lymphocytosis has similarly been shown to be of little diagnostic value.

There are a number of etiologic possibilities to be entertained in this patient. The absence of typical ECG changes essentially precludes viral pericarditis. The presence of a tuberculous effusion should be evaluated by culturing pericardial fluid for Mycobaterium tuberculosis (MTB) and obtaining pericardial fluid adenosine deaminase (ADA) levels. The possibility of a malignant effusion must also be considered and might be detected by fluid cytology. Finally, serum autoantibody titres should be measured to evaluate for systemic lupus erythematosus (SLE).

JBS: Bacterial and mycobacterial cultures of the pericardial fluid ultimately returned negative, as did fluid cytology. An ADA level was not ordered. Serum interferon-gamma release assay for MTB (interferon $\gamma$ release assay (IGRA)-MTB) was likewise negative as was testing for HIV, a comprehensive panel of autoimmune serologies and a urine histoplasma antigen. CT of the chest with intravenous contrast revealed several small ill-defined nodules predominantly in the right upper lobe with calcification of the mediastinal and hilar lymph nodes (figure $1 \mathrm{~F}$ ) as well as the spleen (not shown). There was no evidence of malignancy. Reaccumulation of pericardial fluid prompted referral for a pericardial window. Histology from the surgical specimen was non-diagnostic with negative tissue cultures. The patient's hospital course was further complicated by a minor ischaemic cerebrovascular accident requiring treatment with 

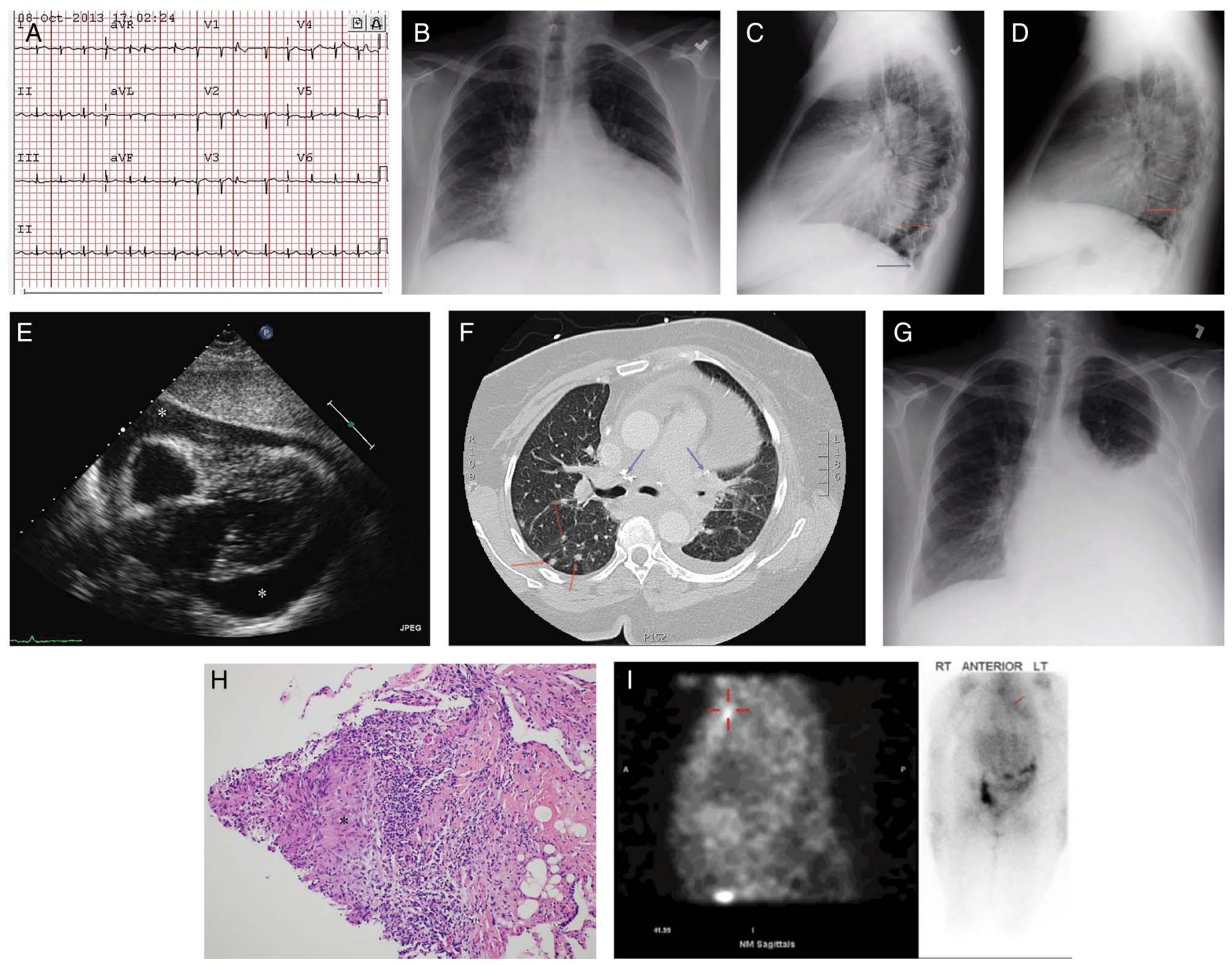

Figure 1 (A) ECG showing sinus tachycardia at $100 \mathrm{bpm}$ with atrial premature complexes and borderline low QRS voltage. (B) Posteroanterior chest radiograph with an enlarged cardiac silhouette and clear lung fields. (C) Lateral chest radiograph with blunting of the costophrenic angles by tiny pleural effusions (blue arrow). Interval enlargement of the cardiac silhouette is best appreciated when this lateral view is compared with that in (D) taken 10 months earlier (red arrow). (E) Subcostal echocardiographic image showing a circumferential pericardial effusion (asterisks).

(F) Chest CT demonstrating right lung nodules (red arrows) as well as intrathoracic lymph node calcifications (blue arrows). (G) Posteroanterior chest radiograph with a large left pleural effusion. $(\mathrm{H})$ Representative transbronchial biopsy specimen from this patient containing a non-caseating granuloma (asterisk) (H\&E, original magnification $\times 200)$. (I) Limited $48 \mathrm{~h}$ Gallium-67 scan showing increased uptake in the left superior mediastinum (red callipers) on a sagittal single photon emission tomography image (left panel) and in the region of the pericardium (arrow) on a spot image (right panel).

clopidogrel. She was discharged home with the plan to defer further invasive procedures until it was deemed safe to hold antiplatelet therapy. Sputum mycobacterial and fungal cultures were collected and pending at the time of discharge.

OE (Respiratory Consultant): As is commonplace, pericardial tissue failed to establish the aetiology of the pericardial effusion. The negative result of the IGRA-MTB further diminishes the already low likelihood of reactivation MTB in a native of the USA. The combination of lymph node and splenic calcifications is usually related to prior granulomatous involvement. Among granulomatous infections endemic to the USA, Histoplasma capsulatum is the most common pathogen, a fungus prevalent in the mid-Western and Southern states. It can disseminate subclinically at the time of initial exposure, leaving behind calcifications in involved organs, and then reactivate years later. Pericarditis with effusion due to $H$. capsulatum has been described but is very rare and unlikely in a lifelong resident of New York State barring exposure during travel. ${ }^{2}$ The negative urine histoplasma antigen and the absence of systemic findings further argues against disseminated histoplasmosis with pericardial disease. It is difficult to classify the distribution pattern of the scant nodularity on this patient's CT scan. Granulomatous infection would be expected to produce a centrilobular or random pattern. A perilymphatic distribution characterised by nodules adjacent to the pleura and fissures, on the other hand, would raise the possibility of sarcoidosis, which could account for organ calcifications as well. The most common clinically apparent sites of involvement in sarcoidosis are the lungs, lymph nodes, skin and eyes. Clinically significant pericardial involvement is very unusual, especially one that leads to tamponade physiology. ${ }^{3}$ The incidental discovery of pericardial granulomas at autopsy and the detection of small, asymptomatic pericardial effusions on routine echocardiography occur in a much larger percentage of sarcoidosis cases. ${ }^{3}$ Interestingly, nearly all of the reported patients with sarcoidosis with massive pericardial effusions have been African-American women. In any event, this would be an extremely uncommon presentation of sarcoidosis. Malignancy has not been conclusively excluded, though it appears to be an 
unlikely explanation. The lack of compatible clinical findings and autoantibodies argues against SLE.

JBS: Approximately a month later the patient was readmitted with dyspnoea on exertion. Chest radiography revealed a large left pleural effusion (figure 1G). Repeat echocardiography showed normal ventricular function and no significant pericardial fluid. All four sputum samples sent for mycobacterial culture on the prior admission were found to be negative for MTB but positive for Mycobacterium avium complex (MAC). Sputum fungal culture had produced no growth. Thoracentesis yielded a lymphocyte-predominant exudative fluid with negative cultures and cytology. The pleural fluid ADA level was 18.6 U/L. The ratio of CD4+/CD8 + lymphocytes in the fluid was $>5$ with no evidence of monoclonality.

OE: One assumes that the pericardial and pleural effusions in this patient share a common aetiology. The low pleural fluid ADA further reduces the likelihood of MTB. MAC infection, which is localised to the lung in immunocompetent hosts, could account for the nodules seen on CT and the pleural effusion but has not been definitively implicated in pericardial disease in the HIV-negative population. Occult malignancy remains a remote possibility, though a second negative cytology lowers the probability. The absence of lymphocyte monoclonality argues against lymphoma. At this juncture, sarcoidosis emerges as an intriguing candidate. Pleural effusions in sarcoidosis are rare, detected in only 5 of 181 outpatients $(2.8 \%)$ in the largest published series. ${ }^{4}$ When sampled, they are usually lymphocytic exudates, and the $\mathrm{CD} 4+/ \mathrm{CD} 8+$ ratio $>1$ mirrors that seen in the bronchoalveolar lavage (BAL) fluid of patients with sarcoidosis. Historically the majority of cases in which histological examination of the pleura was performed have yielded non-caseating granulomas. Symptomatic, large pleural effusions in sarcoidosis are extremely uncommon; in most cases, effusions are incidentally discovered on imaging or at autopsy. ${ }^{4}$ The English-language literature contains only one report of massive pericardial and pleural effusions in the same patient with sarcoidosis. ${ }^{5}$ At this point, histological sampling is needed; options include bronchoscopic biopsy of the lung nodules and percutaneous or thoracoscopic pleural biopsy.

JBS: The patient underwent bronchoscopy with transbronchial biopsy and BAL. The predominant cell type in the fluid was a polyclonal population of lymphocytes with a $\mathrm{CD} 4+/ \mathrm{CD} 8+$ ratio of 2.5. Cytological examination was negative for malignancy. Pathology revealed non-caseating granulomas with giant cells (figure $1 \mathrm{H}$ ). Special stains for microorganisms were negative. Neither tissue nor BAL cultures for acid-fast bacilli (AFB) and fungus were collected by the bronchoscopist.

OE: Although sarcoidosis is a systemic granulomatous disease, in a patient presenting with compatible findings the demonstration of granulomas on biopsy of a single organ or a granulomatous reaction to the Kveim-Siltzbach reagent is considered sufficient for diagnosis. This patient's presentation would be highly unusual for sarcoidosis, so a search for a second confirmatory biopsy site ought to be considered. Additionally, sarcoidosis is a diagnosis of exclusion after competing diagnoses such as infection and malignancy have been firmly ruled out. It would have thus been advisable to obtain AFB and fungal cultures at the time of bronchoscopy. Although the lung granulomas could be attributed to pulmonary MAC infection alone, the other features of this patient's illness, especially the pericardial effusion, mitigate against that theory. Given the serious nature of this patient's presentation with presumed sarcoidosis, treatment with prednisone should be initiated. There is no conclusive evidence that corticosteroid therapy alters the clinical behaviour of pre-existing pulmonary MAC or leads to its dissemination.

JBS: Examination of the lacrimal and parotid glands as well as the exposed skin was normal. Gallium-67 scanning excluding the head due to claustrophobia demonstrated increased mediastinal, hilar, upper lobe and pericardial uptake (figure 1I). The serum calcium level was normal while the ACE level was $67 \mathrm{U} / \mathrm{L}$ (normal range 9-67 U/L). The patient was discharged with a prescription of prednisone $30 \mathrm{mg}$ daily with a provisional diagnosis of sarcoidosis. Outpatient ECG performed for a complaint of palpitations was unremarkable, and 30-day event recording showed only occasional atrial ectopy. Approximately 6 weeks later she presented with progressively worsening alopecia and a previously undetected raised, hyperpigmented scalp lesion in an area of hair loss (figure 2A). Skin biopsy revealed extensive noncaseating granulomatous inflammation (figure 2B). The patient's corticosteroid dose was tapered over the subsequent 12 months. By the end of therapy, her pleural effusion had resolved as had her dyspnoea. The scalp lesion, although decreased in size, persisted despite the prolonged prednisone course.

OE: The granulomatous scalp lesion, discovered incidentally due to alopecia complicating systemic corticosteroid therapy, establishes sarcoidosis as the most likely cause of the massive
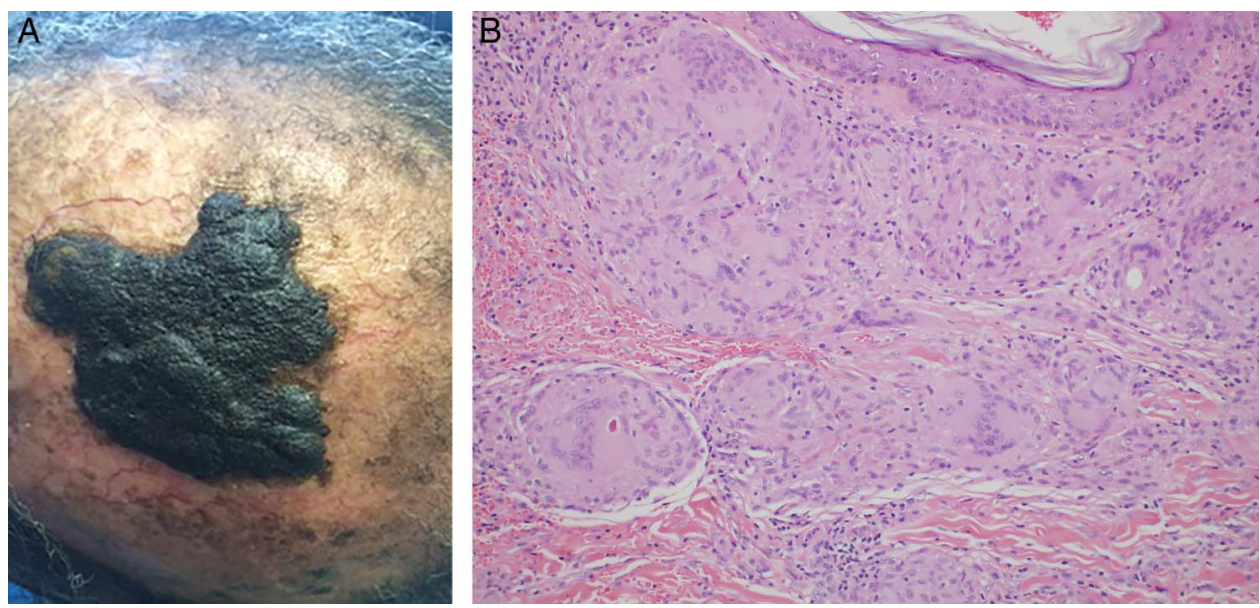

Figure 2 (A) Hyperpigmented, hyperkeratotic plaque on the patient's scalp surrounded by alopecia. (B) Biopsy of the lesion shown in (A) demonstrating extensive non-caseating granulomatous inflammation of the dermis ( $\mathrm{H} \& \mathrm{E}$, original magnification $\times 200)$. 


\section{Chest clinic}

pericardial and pleural effusions in this case. In a patient with suspected sarcoidosis presenting atypically, it is reassuring to recover granulomas from more than one organ site. The fact that her pleural effusion and symptoms completely resolved with prednisone implies that her persistent sputum positivity for MAC likely represented colonisation and further supports sarcoidosis as the unifying aetiology. This is a fascinating case in which a medication side effect helped resolve a diagnostic dilemma while reminding us that clues to the presence of sarcoidosis can hide in unexpected places.

JJS: Cardiac sarcoidosis can cause conduction disturbances and ventricular tachyarrhythmias, neither of which was detected during prolonged monitoring in this patient. The discovery of such abnormalities would have mandated scanning with positron emission tomography or cardiac MRI. ${ }^{3}$ Should inflammatory involvement be detected by these modalities, insertion of an implantable cardioverter defibrillator for primary prevention would merit strong consideration. ${ }^{3}$
Correction notice This article has been corrected since it was published Online First. Figure 1 (I) caption has been corrected.

Competing interests None declared.

Patient consent Obtained.

Provenance and peer review Not commissioned; externally peer reviewed.

\section{REFERENCES}

1 Ben-Horin S, Shinfeld A, Kachel E, et al. The composition of normal pericardial fluid and its implications for diagnosing pericardial effusions. Am J Med 2005;118:636-40.

2 Picardi JL, Kauffman CA, Schwartz J, et al. Pericarditis caused by Histoplasma capsulatum. Am J Cardiol 1976;37:82-8.

3 Ayyala US, Nair AP, Padilla ML. Cardiac sarcoidosis. Clin Chest Med 2008;29:493-508.

4 Huggins JT, Doelken P, Sahn SA, et al. Pleural effusions in a series of 181 outpatients with sarcoidosis. Chest 2006;129:1599-604.

5 Currie GP, Kerr K, Buchan K, et al. A rare cause of recurrent massive pericardial and pleural effusions. Q J Med 2008;101:989-92. 